\title{
Gyrate atrophy of the choroid and retina: 15 Japanese patients
}

\author{
S HAYASAKA,' T SHIONO,' K MIZUNO,' C SASAYAMA, ${ }^{2}$ S AKIYA, ${ }^{3}$ \\ Y TANAKA, ${ }^{3}$ M HAYAKAWA,${ }^{4}$ Y MIYAKE, ${ }^{5}$ AND N OHBA ${ }^{6}$
}

From 'Tohoku University, ${ }^{2}$ Chiba University, ${ }^{3}$ Keio University, ${ }^{4}$ Juntendo University, ${ }^{5}$ Nagoya University, and ${ }^{6}$ Kagoshima University

SUMmARY We examined 15 Japanese patients who had gyrate atrophy of the choroid and retina with hyperornithinaemia. Their visual acuities fell to 0.2 or worse in the second or third decade of life. Myopia developed late in the first decade, and the refractions decreased to -10 or -15 dioptres at age 20 . Tunnel vision developed at approximately age 20 . Our results suggested that the visual functions of Japanese patients were worse in the third decade or later than similarly affected Finnish patients.

Simell and Takki' first described the association of gyrate atrophy of the choroid and retina with hyperornithinaemia in 1973. Since then much attention has been devoted to this rare inherited disorder. So far about 90 biochemically confirmed cases have been reported in the world literature ${ }^{2}$; about one-third of these are Finnish. ${ }^{34}$ We present herein the clinical features of 15 Japanese patients with biochemically confirmed gyrate atrophy and hyperornithinaemia and compare them with similarly affected Finnish patients. Some of our cases have been previously reported elsewhere. ${ }^{5-13}$

\section{Patients and methods}

Letters inquiring about the existence of patients with gyrate atrophy of the choroid and retina were sent to 80 departments of ophthalmology at universities and medical colleges in Japan in 1984. Replies came from 77 institutions. A total of 15 patients with gyrate atrophy and hyperornithinaemia were identified, and all were re-examined by us. One patient who had been receiving proline for six years (reported as case 1 in an earlier report ${ }^{6}$ ) and another whose ornithine level in the serum was reduced with vitamin $\mathbf{B}_{6}$ therapy (case 3 in the same report ${ }^{6}$ ) were particularly analysed.

The visual acuity for all patients was determined with full correction. Refraction was examined with a

Correspondence to Seiji Hayasaka, MD, Department of Ophthalmology, Tohoku University School of Medicine, Sendai, Miyagi 980, Japan. streak retinoscope or refractometer. The anterior segment of the eye was observed by slit-lamp examination, and the posterior segment was examined by indirect ophthalmoscopy, biomicroscopic observation with a Goldmann three-mirror lens, and fluorescein angiography. Visual fields were examined by Goldmann perimetry. The serum or plasma ornithine level was determined with a Hitachi amino acid analyser.

\section{Results}

Of the 15 patients with gyrate atrophy in Japan 11 were male and four were female. Their ages ranged from 9 to 55 years in 1984. All showed yellowish round patches of atrophy in the peripheral fundus or typical chorioretinal atrophy. Characteristic fundus lesions were confirmed by fluorescein angiography.

Fig. 1 shows the visual acuities of both eyes of each patient according to age. Until age 15 the patients had relatively good central vision, from 0.2 to $1 \cdot 0$, if vitreous haemorrhage had not occurred. The central vision of eyes after vitreous haemorrhage decreased to hand motion or light perception. Visual acuity reduced to $\mathbf{0 . 2}$ or worse was found in the second or third decade. Cataract extraction was performed in the third or fourth decade. After cataract extraction the visual acuity increased to various levels (range, 0.03 to 0.6 ). The subsequent visual impairment was dependent on macular involvement. The patient who received proline for six years ${ }^{6}$ had central vision of 1.0 at age 10 . The patient who received vitamin $B_{6}$ 


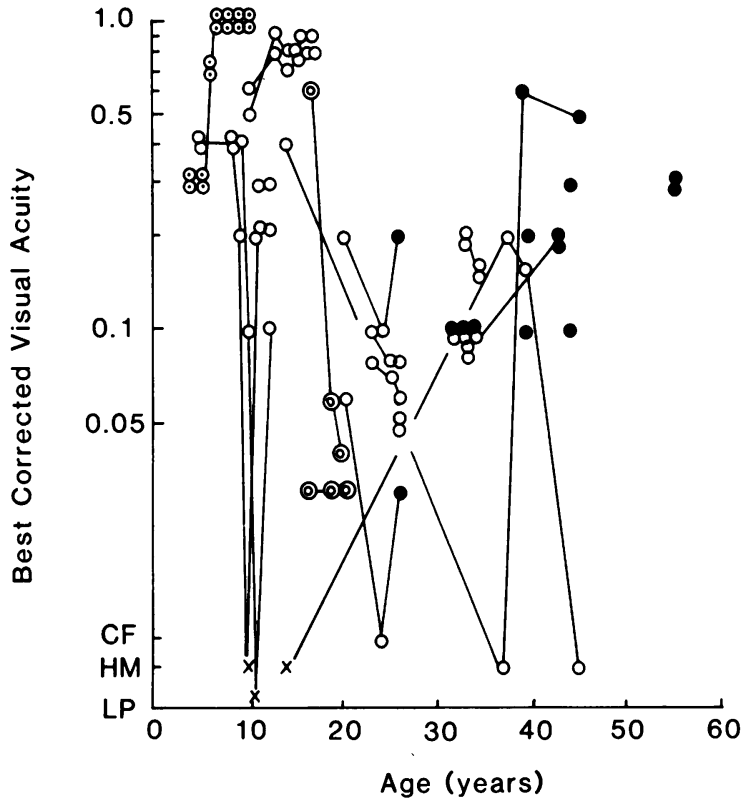

Fig. 1 Visual acuities of 30 eyes of patients with gyrate atrophy of the choroid and retina. O Phakia; aphakia; $\times$ vitreous haemorrhage; O proline supplemented case; (C) vitamin $B_{6}$ responsive case.

showed reduced central vision in spite of the therapy.

Fig. 2 shows the refractions of 21 phakic eyes by age. Myopia developed late in the first decade, and refractions declined to -10 or -15 dioptres by age

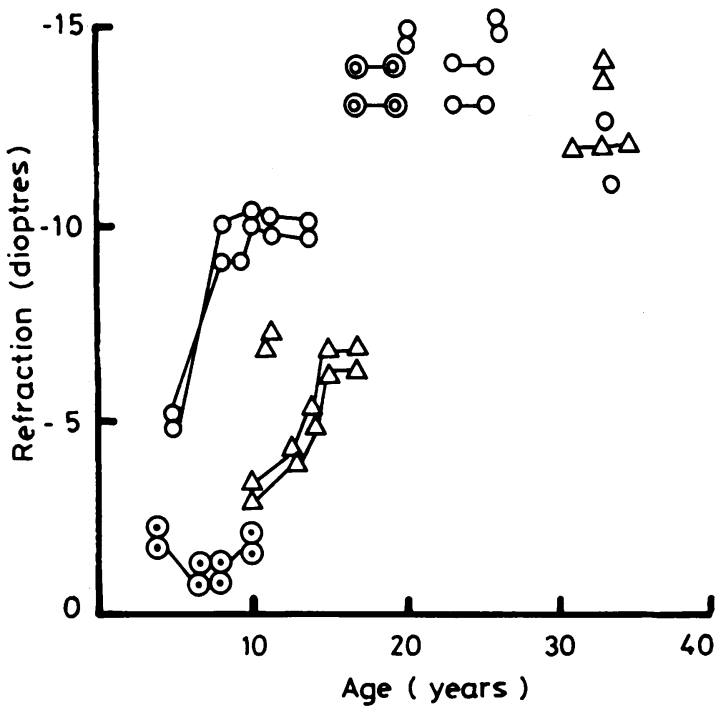

Fig. 2 Refractions of 21 phakic eyes of patients with gyrate atrophy of the choroid and retina. $\bigcirc$ male; $\triangle$ female; Oproline supplemented case; (O) vitamin $B_{6}$ responsive case.

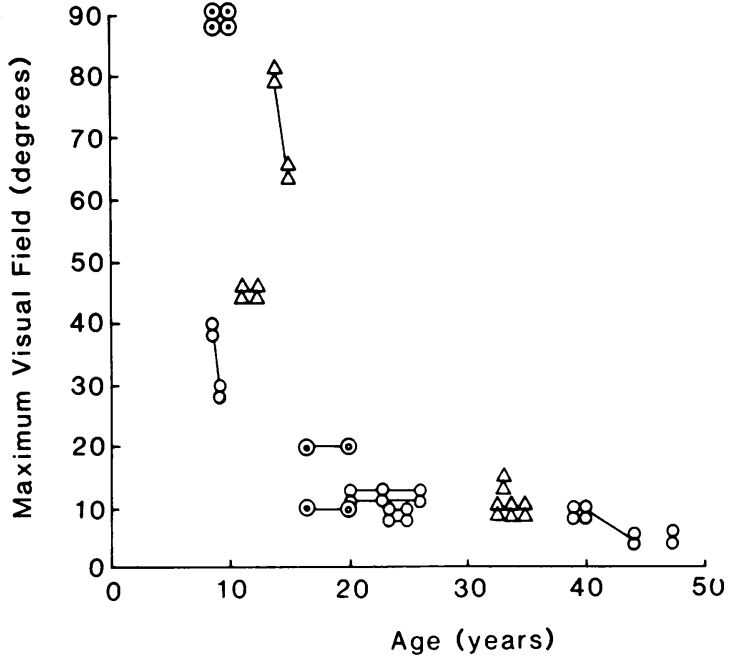

Fig. 3 Maximum visual fields of 24 eyes of patients with gyrate atrophy of the choroid and retina. O male; $\triangle$ female; O proline supplemented case; $\bigcirc$ vitamin $B_{6}$ responsive case.

20. The patient who received proline had the smallest refractive error. The vitamin $B_{6}$ responsive patient experienced no change in refraction due to the vitamin therapy.

Fig. 3 demonstrates the decreasing of visual fields with age in 24 eyes. Tunnel vision developed at approximately age 20 . No difference in visual field was noted between males and females. The visual

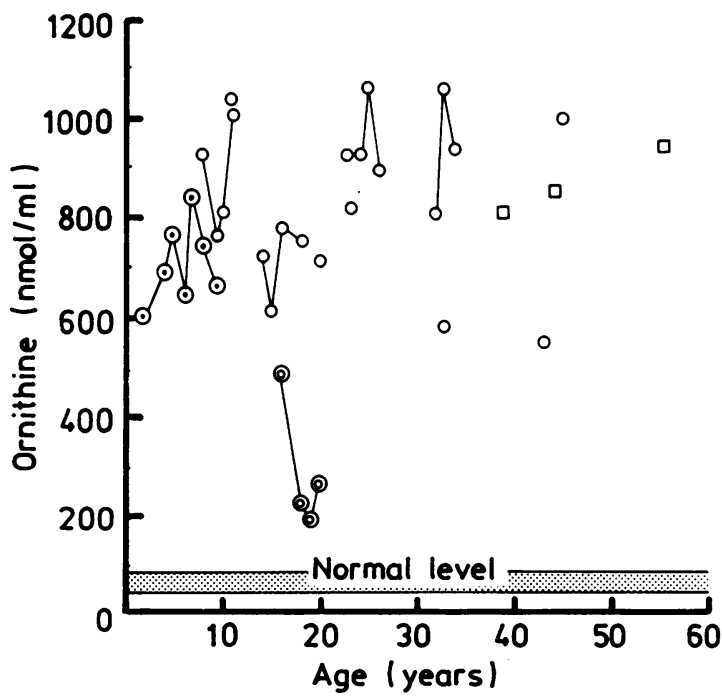

Fig. 4 Serum or plasma ornithine levels in 15 patients with gyrate atrophy of the choroid and retina. O serum; $\square$ plasma; Oproline supplemented case; (O) vitamin $B_{6}$ responsive case. $(\mathrm{nmol} / \mathrm{ml}=\mathrm{mmol} / \mathrm{l}$.) 
fields of the patient who received proline were full. The vitamin $\mathrm{B}_{6}$ responsive patient showed no change in visual field from the therapy.

Fig. 4 shows the serum or plasma ornithine levels in 15 cases. The ornithine levels, which ranged from 500 to $1100 \mathrm{mmol} / \mathrm{l}$ in patient on a regular diet, did not change with age. The serum ornithine levels in the patient receiving proline were unchanged. In the vitamin $B_{6}$ responsive patient the serum ornithine level decreased with oral administration of the vitamin, as shown previously. ${ }^{5}$

Of the 15 cases, parental consanguinity was present in eight, absent in four, and unconfirmed in three.

\section{Discussion}

Patients were identified from the replies received from our letters of inquiry. Although all Japanese patients were not included in this present study, it is possible that nearly all cases of gyrate atrophy might have been represented.

Takki and Milton reported on the natural history of 27 Finnish patients with gyrate atrophy. ${ }^{4}$ Differences in central and peripheral vision were noted between their Finnish and our Japanese patients. The central vision in phakic Japanese patients at age 20 or later was below $0 \cdot 2$, or worse than that in the Finnish patients. François observed that 'visual acuity may remain rather good for a long time' in his review of gyrate atrophy ${ }^{14}$ It is likely that his data were based on reports excluding Japanese cases. Severe constriction of visual fields developed in the Japanese patients at about age 20 , while tunnel vision proceeded in the Finnish patients to the fourth or fifth decade. ${ }^{4}$ Takki and Milton ${ }^{4}$ showed that some women retained larger visual fields. The Japanese patients showed no difference in the deterioration of peripheral vision between males and females. Possibly the visual functions of Japanese patients with gyrate atrophy in the third decade or later were more deteriorated than those of Finnish patients. However, why deficient activity of ornithine-ketoacid-transaminase has the different visual effects remains obscure.

The patient who received proline for six years showed good central and peripheral vision and mild myopia. The vitamin $B_{6}$ responsive patient, however, had no improvement in vision, though the therapy reduced the patient's serum ornithine level.

The reason for a predominance of male patients
(11 to 4) in our series was unknown. Consanguinity of the patients' parents was found in half the cases, suggesting a recessive trait.

The authors would like to thank the 77 departments of ophthalmology at universities and medical colleges in Japan for replying to our inquiry. We also thank Ms M Gere for editing the manuscript. This study was supported in part by grants from the Ministry of Education and the Ministry of Health and Welfare, Japan.

\section{References}

1 Simell O, Takki K. Raised plasma ornithine and gyrate atrophy of the choroid and retina. Lancet 1973; i: 1031-3.

2 Valle D, Simell O. The hyperornithinemias. In: Stanbury JB, Wyngaarden JB, Fredrickson DS, Goldstein JL, Brown MS, eds. The metabolic basis of inherited disease. 5th ed. New York: McGraw-Hill, 1983: 382-401.

3 Takki K. Gyrate atrophy of the choroid and retina associated with hyperornithinemia. Br J Ophthalmol 1973; 58: 3-23.

4 Takki K, Milton RC. The natural history of gyrate atrophy of the choroid and retina. Ophthalmology (Rochester) 1981; 88: 292-301.

5 Hayasaka S, Saito T, Nakajima H, et al. Gyrate atrophy with hyperornithinaemia: different types of responsiveness to vitamin B $_{6}$. Br J Ophthalmol 1981; 65: 478-83.

6 Hayasaka S, Saito T, Nakajima H, Takahashi O, Mizuno K, Tada K. Clinical trials of vitamin $B_{6}$ and proline supplementation for gyrate atrophy of the choroid and retina. Br J Ophthalmol 1981; 69: 283-90.

7 Nakajima H, Hayasaka S, Shiono T, et al. A case of gyrate atrophy of the choroid and retina associated with hyperornithinemia. Jpn J Ophthalmol 1981; 25: 495-500.

8 Takahashi O, Hayasaka S, Kiyosawa M, et al. Gyrate atrophy of choroid and retina complicated by vitreous hemorrhage. Jpn J Ophthalmol 1985; 29: 170-6.

9 Hayasaka S, Shoji K, Kanno C, Oura F, Mizuno K. Differential diagnosis of diffuse choroidal atrophies: diffuse choriocapillaris atrophy, choroideremia, and gyrate atrophy of the choroid and retina. Retina 1985; 5: 30-7.

10 Akiya S, Ohsawa M, Ogata T. The long-term observation of two brothers of gyrate atrophy of the choroid and retina with hyperornithinemia (in Japanese). Nippon Ganka Gakkai Zasshi 1977; 81: 310-22.

11 Nakajima A, Hayakawa M, Kitagawa T. Study on hyperornithinemia with gyrate atrophy. I. (In Japanese.) Annual report on retinochoroidal atrophy study. Ministry of Health and Welfare, Japan: 1981: 106-10.

12 Sakiyama T, Kitagawa T, Hayakawa M. Study on hyperornithinemia with gyrate atrophy. II. (In Japanese.) Annual report on retinochoroidal atrophy study. Ministry of Health and Welfare, Japan: 1982: 125-8.

13 Ota I, Miyake Y, Ichikawa $\mathrm{H}$. A family with gyrate atrophy of the choroid and retina. (In Japanese.) Jpn Rev Clin Ophthalmol 1985; 79: 1221-3.

14 François J. Gyrate atrophy of the choroid and retina. Ophthalmologica 1979; 178: 311-20.

Accepted for publication 5 December 1985. 